\title{
Posterior Cingulate and Lateral Parietal Gray Matter Volume in Older Adults with Depressive Symptoms
}

\author{
Michele L. Ries • Allison Wichmann • \\ Barbara B. Bendlin • Sterling C. Johnson
}

Received: 8 July 2008 / Accepted: 11 March 2009 /Published online: 15 April 2009

C) The Author(s) 2009. This article is published with open access at Springerlink.com

\begin{abstract}
Depressive symptoms occurring late in life are an important risk factor for Alzheimer's disease (AD). The latest research finds that onset of depressive symptoms in late life may herald the development of $\mathrm{AD}$, not only for amnestic Mild Cognitive Impairment (aMCI) patients, but also for cognitively-normal older adults. Neuroimaging of brain structure, blood flow, and glucose metabolism indicates that depressive symptoms in late life are accompanied by structural and functional changes in limbic brain regions vulnerable to $\mathrm{AD}$. The present cross-sectional study was guided by the hypothesis that compared to their nondepressed counterparts, older adults with mild to moderate depressive symptoms have less volume in limbic structures vulnerable to changes in AD - specifically, cortical midline structures such as anterior cingulate and posterior cingulate cortex as well as mesial temporal regions such as bilateral hippocampi and amygdalae. Consistent with our hypothesis, results of a voxel-based morphometry analysis revealed smaller retrosplenial, posterior cingulate, and precuneus gray matter volumes in depressed individuals relative to healthy controls. Right lateral parietal cortex-another region vulnerable to change in $\mathrm{AD}$ - was also smaller in the group with depressive symptoms. Contrary to our hypothesis, no volumetric differences were found in the anterior cingulate cortex or mesial temporal lobe. Results of this study show a relationship between geriatric depressive
\end{abstract}

M. L. Ries $(\bowtie) \cdot$ A. Wichmann • B. B. Bendlin • S. C. Johnson Geriatric Research Education and Clinical Center, William S. Middleton Memorial Veterans Hospital,

Madison, WI 53705, USA

e-mail: mlr@medicine.wisc.edu

M. L. Ries • A. Wichmann • B. B. Bendlin • S. C. Johnson University of Wisconsin-Madison,

Madison 53705, USA symptoms and brain volume in regions vulnerable to AD. Follow-up of participants over time will tell if brain changes detected here predict development of AD.

Keywords Aging - Alzheimer disease - Depression · Magnetic resonance imaging $\cdot$ Affect

With the expected upsurge in the incidence and prevalence of Alzheimer's disease (AD) in coming decades, increasing research attention focuses on early detection (Fagan et al. 2005; Mueller et al. 2005; Sager et al. 2005). A handful of important AD risk factors are known-including specific genotypes such as homo- or heterozygosity for the apolipoprotein $4(\mathrm{APO} \varepsilon 4)$ allele, family history of $\mathrm{AD}$, and amnestic mild cognitive impairment (aMCI) — a condition in which one shows impaired memory but intact functional abilities. Depressive symptoms occurring late in life are also an important risk factor. In fact, some reports suggest that affective changes may be the earliest symptoms of AD pathology in some individuals - even predating the cognitive decline that portends development of AD (Geda et al. 2006; Stepaniuk et al. 2008).

Neuropsychiatric symptoms are more prevalent in AD than in the rest of the elderly population, with $35-75 \%$ of AD patients showing depressive symptoms, apathy, anxiety, or increased irritability (see Apostolova and Cummings 2008 for review). Prevalence estimates of neuropsychiatric symptoms vary with the source of the study sample, the sensitivity of the neuropsychiatric measure used, and the use of a formal psychiatric interview versus symptom questionnaire-yet depressive symptoms and apathy are consistently the most common neuropsychiatric symptoms in mild AD. (In our discussion of the literature, a formal diagnosis of Major Depressive Disorder ascertained by a structured psychiatric interview will be indicated as MDD; 
depressive symptoms otherwise assessed that do not meet formal DSM-IV diagnostic criteria for MDD are denoted as "depression" or depressive symptoms.) Individuals with aMCI show a high prevalence MDD, and presence of MDD in aMCI introduces a 2.6-fold increase for developing AD over the next three years (Modrego and Ferrandez 2004). Furthermore, depressive symptoms shown by many aMCI patients who progress to $\mathrm{AD}$ is refractory to anti-depressant medications (Li et al. 2001).

Symptoms in many depressed older patients who later develop $\mathrm{AD}$ may have a different etiology than depressive symptoms in younger adults - this etiology may be AD pathology or a factor mediating the association between depression and $\mathrm{AD}$. AD with comorbid depressive symptoms is associated with greater cortical neurofibrillary tangle development than AD alone (Rapp et al. 2008). Also, studies of late-life depression that control for anti-depressant use and cardiovascular risk found that compared to non-depressed older adults, their depressed counterparts have lower plasma $\mathrm{A} \beta_{42}$ levels and higher $\mathrm{A} \beta_{40}: A \beta_{42}$ ratios - a profile similar to that seen in many early AD patients (Qiu et al. 2007; Sun et al. 2008). Additionally, recent research finds that onset of depressive symptoms in late life may herald the development of $\mathrm{AD}$, not only for aMCI patients, but also for cognitivelynormal older adults (Geda et al. 2006; Stepaniuk et al. 2008). Taken together, these new data suggest that in some older adults, late-onset depression is an early behavioral sign of AD.

Neuroimaging of brain structure, blood flow, and glucose metabolism indicates that depressive symptoms in late life relate to structural and functional changes in limbic brain regions vulnerable to $\mathrm{AD}$. Volumetric studies using region of interest (ROI) analyses show that older adults with depressive symptoms show reduced hippocampal, anterior cingulate, and orbitofrontal volumes compared to non-depressed controls (Ballmaier et al. 2004; BellMcGinty et al. 2002; Drevets et al. 1997); however, these findings are not unequivocal (Kumar et al. 2006). Anterior and posterior cingulate cortex and the amygdala also show abnormal glucose metabolism and blood flow in MDD (Bench et al. 1992; Drevets 1999; Drevets et al. 1997).

The numerous ROI volumetric brain studies cited above have advanced our understanding of brain volume changes particularly hippocampal changes - in late-life depression. However, much is to be learned about the wider-range of brain changes occurring in geriatric depression. Changes in brain structures that have less clearly defined boundaries are difficult to quantify with ROI analysis, and for this reason these brain regions are not typically studied via ROI methods. In contrast to region-specific analyses, voxel-based morphometry (VBM) provides a comprehensive assessment of anatomical differences throughout the brain, avoiding possible bias inherent in investigating volume differences within only selected brain structures.
The aim of the current cross-sectional VBM study is to investigate volumetric differences of limbic brain regions in healthy elderly adults with and without depressive symptoms. Many studies cited above have investigated symptoms of major depressive disorder; however, others have found that subclinical depressive symptoms are also prognostic of MCI and AD. We hypothesized that older adults with depressive symptoms have less volume in limbic structures vulnerable to changes in $\mathrm{AD}$, specifically cortical midline structures such as anterior cingulate and posterior cingulate cortex as well as mesial temporal regions such as bilateral hippocampi and amygdalae.

\section{Methods}

\section{Participants}

Forty-seven cognitively-healthy elderly individuals in the Madison community who responded to newspaper advertisements and mass e-mails to the University of Wisconsin-Madison community for studies on aging and cognition participated in this study. Participants whose data are included in this manuscript were recruited as cognitively-healthy control participants. At entry into this study, subjects were asked if they had a history of or current Major Depression that required treatment. Indicating a positive history for treated Major Depression was exclusionary for this study. Depressive symptoms were measured via the Center for Epidemiologic Studies Depression (Radloff 1977; CES-D). The CES-D includes twenty items rating somatic, evaluative, mood, and motivational symptoms of depression experienced within the past week. Symptoms targeted by each item are rated on a four-point frequency scale (i.e., rarely/none, some/ little, occasionally/moderate, most/ all of the time). Items on the CES-D load onto four factors: depressed affect, positive affect, somatic symptoms/retarded activity, and interpersonal activity. Fifteen participants comprising a "depressed" group endorsed mild to moderate depressive symptoms as measured by the CES-D (score $\geq 10$ ). None of the participants were being treated for major depressive disorder or had a history of major depressive disorder according to their self-report. This group with depressive symptoms is labeled the "depressed group" in this paper. Thirty-two participants comprising a control group endorsed no or minimal symptoms of depression (score $\leq 5$ ). The two groups were similar in age, gender distribution, and performance on several neuropsychological tests (see Table 1). The control group had significantly more education than the depressed group. Therefore, this variable was included as a covariate within the statistical model. 
Table 1 Demographic and Neuropsychological Data for depressed and non-depressed groups

\begin{tabular}{|c|c|c|}
\hline & Depressed $(n=15)$ & Non-depressed $(n=32)$ \\
\hline Age & $66.3(5.3)$ & $68.4(7.4)$ \\
\hline Gender(M:F) & $5: 10$ & $14: 18$ \\
\hline Education & $14.6(2.4)$ & $17.3(2.3)$ \\
\hline CES-D ${ }^{\mathrm{a}}$ & $17.3(6.5)$ & $1.8(1.8)$ \\
\hline Trails A & $30.1(7.4)$ & $32.3(12.7)$ \\
\hline Trails B & $67.9(18.3)$ & $71.5(26.1)$ \\
\hline $\mathrm{MMSE}^{\mathrm{b}, \mathrm{g}}$ & $29.0(1.0)$ & 29.2(1.9) \\
\hline RAVLT $^{\mathrm{c}}$ Total & $47.0(8.1)$ & $47.1(7.8)$ \\
\hline RAVLT $^{\mathrm{c}}$ Delay Recall & $9.3(2.6)$ & $9.0(2.7)$ \\
\hline BVMT $^{\mathrm{d}}$ Total & $25.7(6.9)$ & $24.7(7.0)$ \\
\hline BVMT $^{\mathrm{d}}$ Delay Recall & $9.4(2.4)$ & $9.6(2.6)$ \\
\hline COWAT $^{\mathrm{e}, \mathrm{g}}$ & $43.3(11.9)$ & $47.0(11.8)$ \\
\hline Animal Fluency ${ }^{\mathrm{g}}$ & $21.6(5.1)$ & $20.7(4.3)$ \\
\hline
\end{tabular}

${ }^{\text {a }}$ Center for Epidemiologic Studies Depression

${ }^{\mathrm{b}}$ Mini Mental State Examination

${ }^{\mathrm{c}}$ Rey Auditory Verbal Learning Test

${ }^{\mathrm{d}}$ Brief Visuospatial Memory Test-Revised

${ }^{\mathrm{e}}$ Controlled Oral Word Associated Test

${ }^{\mathrm{f}}$ All indices of test performance are raw scores

${ }^{\mathrm{g}}$ Number of participants included in mean and standard deviation calculations MMSE 14:32, COWAT 13:28, Animal Fluency 11:30

All subjects were cognitively normal. Exclusion criteria for both groups included cognitive disorders such as mild cognitive impairment (MCI) or AD, a history of traumatic brain injury defined by traumatically induced loss of consciousness for greater than ten minutes, diagnosis of other neurological disorder including multi-infarct dementia, alcohol and drug abuse or dependence, Hachinski Ischemia Scale $\leq 4$, hypertension with persistent blood pressure recordings on at least three occasions of diastolic $>$ 100 ; systolic $>160$, MRI incompatibility, diagnosis of major medical disorders including cancer within five years of the study, and participant report of current or prior diagnosis of any psychiatric disorder, including major depressive disorder. Exclusion criteria also included evidence of focal lesions, multiple lacunes, or at least one lacune in a critical memory structure on the T1-weighted MRI scan. All subjects provided written informed consent before participating in this local IRB approved study. Subjects were recruited from the community by advertisement, mailings, and community outreach events.

Neuropsychological assessment

The participants completed a cognitive battery (Table 1) using standardized administration procedures (Spreen and Strauss 1998). The battery examined a variety of functions including verbal and visuospatial learning, memory recall and recognition, and executive functions. There were no significant differences between groups in any of these cognitive measures.

Imaging protocol

Participants were situated on the bed of a GE long bore 3.0 Tesla MRI scanner (General Electric, Milwaukee, WI). Head movement was constrained by foam padding. A 3D inversion recovery-prepared fast gradient echo pulse sequence provided high-resolution T1-weighted structural images acquired in the axial plane with the following parameters: inversion time $=600 \mathrm{~ms}$, fast gradient echo read-out with TR/TE/flip $=9 \mathrm{~ms} / 1.8 \mathrm{~ms} / 20^{\circ}$; acquisition matrix $=256 \times 192 \times 124$ (interpolated to $256 \times 256 \times 124$ ); field of view $=240 \mathrm{~mm}$; slice thickness $=1.2 \mathrm{~mm}(124$ slices); $\pm 16 \mathrm{kHz}$ receiver bandwidth. A Fast Recovery Fast Spin Echo 2D T2-weighted axial sequence was also acquired with the same start and stop locations as the T1 weighted images. The parameters were: field of view $=$ $240 \mathrm{~mm}$, matrix $256 \times 256, \mathrm{TR}=9,000 \mathrm{~ms}, \mathrm{TE}=93 \mathrm{~ms}$, flip angle $=90$. Seventy slices were acquired; slice thickness $=1.7 \mathrm{~mm}$ with $0.3 \mathrm{~mm}$ skip.

A neuroradiologist viewed the anatomical images from each participant for clinically relevant brain abnormalities inconsistent with control diagnosis or requiring clinical follow-up. The T1-weighted images were then used for the VBM analyses.

Voxel-based morphometry methods

The Voxel Based Morphometry (VBM) approach as described by Good et al. (2001) was used to analyze the T1 weighted anatomical images. Statistical Parametric Mapping (SPM5) software was utilized to segment the images into gray matter (GM), white matter (WM), and cerebrospinal fluid (CSF) probability maps. A customized GM template in the common MNI atlas space was created using the T1 volumes of all subjects using methods previously described (Trivedi et al. 2006). The individual GM images were then normalized to this template and smoothed to $12 \mathrm{~mm}$.

Study design and statistical analysis

This was a cross-sectional study comparing the brain volumes of participants with depressive symptoms to those of participants without depressive symptoms. We used an ANCOVA design with gender, education, and total intracranial volume (TICV) as covariates. Due to the relatively small sample sizes, and because our analyses were guided by a priori hypotheses, between-group differences were 


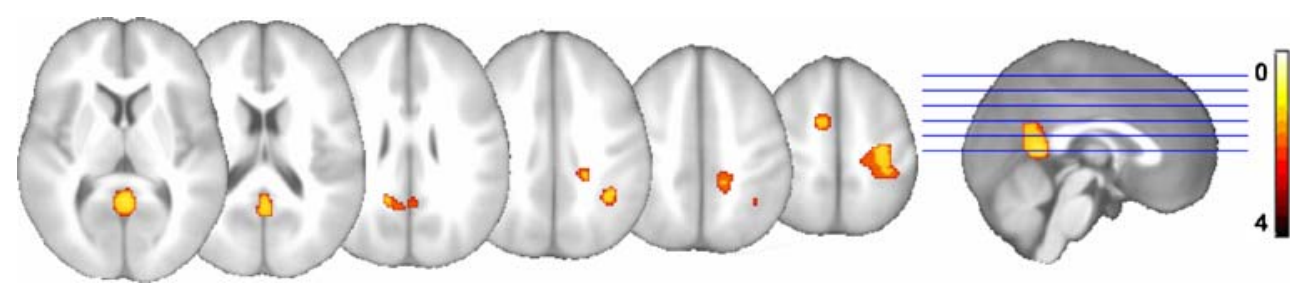

Fig. 1 Results of a two-group t-test revealed that the PCC and right lateral parietal regions had less volume in the depressed group than controls. Lateral and Transverse maximum intensity projections of the entire whole-brain map are also shown. The right side of the brain is on the right side in the images

examined using uncorrected $p$-values (threshold set at $p<.005$, uncorrected).

\section{Results}

\section{Behavioral data}

There were no significant differences between the groups on age or a series of cognitive tests $(p>0.05)$ as summarized in Table 1. Chi squared analysis revealed no significant differences between the groups on gender distribution. The depressed group had significantly fewer years of education than the non-depressed group.

We examined the degree to which participants in the depressed group endorsed items loading on the four factors of the CES-D: depressed affect, positive affect, and somatic symptoms/retarded activity, and interpersonal difficulty. Results of a one-way repeated-measures ANOVA showed significantly different levels of endorsement for the four factors, $F(3,42)=8.08, p<0.001$. Post-hoc t-tests revealed that participants in the depressed group endorsed significantly fewer symptoms indicating interpersonal difficulty than symptoms of depressed affect, $t(14)=4.13, p<.01$, positive affect, $t(14)=3.30, p<.01$, or somatic symptoms/ retarded activity, $t(14)=3.67, p<.01$. There were no significant differences in endorsement of items loading on depressed affect, positive affect, and somatic symptoms/ retarded activity factors.

Imaging data

The VBM analysis tested for group differences in the presence of covariates for gender, education and TICV. The analysis revealed lower gray matter volume in several regions in depressed individuals relative to healthy controls, as depicted in Fig. 1 and Table 2. These included retrosplenial cortex, posterior cingulate cortex, precuneus and lateral parietal areas. There were no volumetric differences in the mesial temporal lobe. Non-depressed participants showed no areas of lower gray matter volume than participants with depressive symptoms.

\section{Discussion}

Recent studies note a significant relationship between depressive symptoms in older adults and increased risk for AD. Therefore, the current study was guided by the hypothesis that older adults with depressive symptoms have less gray matter in cortical midline and mesial temporal limbic regions known to be both associated with affective self appraisal and vulnerable to changes in $\mathrm{AD}$. To test this hypothesis, we used a cross-sectional design to assess gray matter volume differences between elderly individuals with and without depressive symptoms.

Consistent with our hypothesis, results showed that older adults with depressive symptoms (CES-D $\geq 10$ ) had less PCC volume compared with individuals without depressive symptoms (CES-D $\leq 5$ ). The PCC (Vogt et al. 2006)-a region that shows some of the earliest metabolic, function$\mathrm{al}$, and structural changes in individuals at risk for $\mathrm{AD}$ - is part of a network of limbic brain structures involved in memory (Buckner 2004; Morris et al. 2000; Shannon and Buckner 2004), affect (Vogt et al. 2006), and self appraisal (Johnson et al. 2002; Johnson et al. 2006; Northoff et al. 2006). Although the PCC is a limbic region, it is not typically implicated in depressive symptoms of younger adults. However, cognitive-affective appraisals commonly

Table 2 Results of two-group t-test: Non-depressed $>$ Depressed

\begin{tabular}{|c|c|c|c|c|c|c|}
\hline \multirow[t]{2}{*}{ Region } & \multicolumn{3}{|c|}{ MNI coordinates } & \multirow{2}{*}{$\begin{array}{l}\text { Peak } \\
\mathrm{T} \\
\text { value }\end{array}$} & \multirow[t]{2}{*}{$\mathrm{k}$} & \multirow{2}{*}{$\begin{array}{l}\mathrm{P} \\
\text { value }\end{array}$} \\
\hline & $\mathrm{x}$ & $\mathrm{y}$ & $\mathrm{z}$ & & & \\
\hline \multirow[t]{3}{*}{ Lateral Parietal Cortex } & 32 & -48 & 70 & 4.04 & 343 & $>0.001$ \\
\hline & 28 & -20 & 58 & 3.21 & & 0.001 \\
\hline & 32 & -36 & 60 & 3.37 & & 0.001 \\
\hline \multirow{4}{*}{$\begin{array}{l}\text { Posterior Cingulate } \\
\text { Cortex/Retrosplenial } \\
\text { Cortex }\end{array}$} & 2 & -50 & 10 & 3.60 & 344 & $>0.001$ \\
\hline & -16 & -50 & 26 & 3.11 & & 0.002 \\
\hline & -10 & -54 & 30 & 2.90 & & 0.003 \\
\hline & 16 & -38 & 44 & 3.48 & 61 & 0.001 \\
\hline \multirow[t]{2}{*}{ Lateral Parietal Cortex } & 38 & -50 & 40 & 3.30 & 85 & 0.001 \\
\hline & -8 & -2 & 56 & 3.07 & 24 & 0.002 \\
\hline
\end{tabular}


evoke fMRI activity in the PCC in cognitively healthy adults, and depression is characterized by altered selfappraisal. People with depressive symptoms and MDD show predominant negative self-appraisal—a bias indicated by symptoms of worthlessness, pessimism and low selfesteem, and/or they demonstrate apathy — a lack of affective engagement that is necessary to make accurate selfappraisals. Taken within this context, our results suggest that although the PCC is not typically susceptible to changes associated with depression in young adults, changes to the PCC in older adults may constitute a qualitatively-distinct etiology of depressive symptoms. The fact that PCC structure and function is altered in AD and in adults with high AD-risk suggests that this etiology may be AD pathology.

We also found lower gray matter volume in the right lateral parietal cortex of older adults with depressive symptoms. Although this region was not included in our $a$ priori hypothesis, this region shows reliable functional connectivity with the PCC (Greicius et al. 2003; Raichle et al. 2001), and like the PCC, fMRI studies of memory and self-appraisal consistently evoke activity in lateral parietal regions (Northoff et al. 2006; Shannon and Buckner 2004). Furthermore, lateral parietal structure and function is altered in people with $\mathrm{AD}$ and those at risk for developing $\mathrm{AD}$. This supports our contention that AD-like changes may underlie depressive symptoms in many older adults.

Because we chose to conduct whole-brain VBM analysis, we detected parietal brain differences not previously reported in ROI studies that have primarily focused on the mesial temporal lobe. However, our choice of analysis may not have been sensitive enough to detect the hippocampal volume or morphologic differences shown by other studies. Although evidence for hippocampal volume loss in geriatric MDD and depression generated from ROI volumetric studies is mixed (Ashtari et al. 1999; Greenwald et al. 1997; Hickie et al. 2005; Steffens et al. 2000), detection of hippocampal involvement in geriatric depression is seen more consistently when using high resolution MR analysis of hippocampal morphology. A recent study that selectively assessed hippocampal morphology in cognitively-healthy adults found changes specific to the subiculum and CA1 region in older adults with initial depressive symptom development after age 60 (Ballmaier et al. 2008). In a separate study, these same hippocampal regions-the subiculum and CA1 - showed morphologic alterations in MCI patients who developed AD over a three-year period (Apostolova et al. 2006). Our finding of PCC and lateral parietal volume differences, taken with results of studies targeting nuanced hippocampal changes, support the idea that subtle $\mathrm{AD}$-like changes underlie depressive symptoms shown by some older adults.
Our failure to find volumetric group differences in the anterior cingulate cortex was surprising, given that this brain region is commonly implicated in geriatric depression and MDD (Alexopoulos et al. 2008; De Asis et al. 2003). There are several possible reasons for our null result in the ACC. It may be that volume loss in the PCC (a region that possesses both structural and functional connections with the ACC) and lateral parietal lobe shown by some elders with depression precedes ACC volume loss. Another possibility is that our chosen measurement of depressive symptoms - the CES-D — was not sensitive to the aspects of depression typically associated with changes in the ACC and adjacent medial prefrontal cortex. With a measurement scale more sensitive to depressive symptoms such as apathy and reduced motivation and interest-symptoms often associated with ACC changes-we may have detected group differences in this brain region.

Although the current findings are intriguing, conclusions are preliminary due to limitations of our methods. We evaluated depressive symptoms using a validated questionnaire; however, use of a comprehensive psychiatric interview would have allowed better characterization of depressive symptomatology, the extent of depressive symptoms, and the time of onset of depressive symptoms. An analysis that parsed items loading on previously-identified factors revealed that our participants with depressive symptoms endorsed mood symptoms and somatic symptoms, but few items assessing interpersonal difficulty. However, we were unable to make other useful distinctions (e.g., parsing dysphoric feelings versus apathy; or parsing early versus late-life onset). Also, because our sample was communitybased and the range of depressive symptoms was restricted (participants reported not having a psychiatric diagnosis of Major Depressive Disorder), our results can only be generalized to older individuals experiencing mild to moderate depressive symptoms. Individuals who volunteer to participate in clinical research studies often differ from non-volunteers in systematic ways (e.g., higher socioeconomic status; Szklo and Nieto 2006); and this restricts our ability to draw inference about the wider population. In terms of our imaging methods, our choice to conduct a whole-brain, voxel-wise analysis may have forestalled our ability to detect nuanced regional brain alterations - such as those in the hippocampus. Despite these limitations, results of this study show that geriatric depression is associated with decreased brain volume in regions vulnerable to $\mathrm{AD}$. This suggests that the depressed group may have early brain changes associated with Alzheimer's disease; however, this conclusion is preliminary given the cross-sectional design of our study. More definitive assessment of the hypothesis that brain changes seen in depressed older individuals reflect early $\mathrm{AD}$ awaits testing in a prospective cohort study. 
A note about the multifactorial etiology of depressive symptoms provides another caveat to interpreting the current findings. Depressive symptoms and MDD, of course, arise not simply from disease-related changes in neural structure and function, but also as a response to life transitions, disability, and loss (Alexopoulos 2005). Furthermore, the relationship existing between depression and AD-like brain changes is far from simple. Geda et al. (2006) astutely proposed that multiple mechanisms may underlie the association between incident MCI and depression, and similarly, multiple mechanisms may underlie the association between observed AD-like brain changes and depression: (1) AD-like brain changes may cause depression; (2) a separate genetic or environment factor may cause both AD-like brain changes and depression, or (3) brain changes associated with $\mathrm{AD}$ may give rise to depression only in the presence of a mediating genetic susceptibility or environmental factor. It is clear that early detection of $\mathrm{AD}$ will be facilitated through continued investigation of the relationship among the behavioral manifestations of geriatric depression, the brain changes associated with the $\mathrm{AD}$ pathological process, and factors that may mediate this association.

Acknowledgements This study was supported by AG021155 (SCJ). The research was greatly facilitated by Dr. Ries' involvement in the University of Wisconsin Institute for Clinical and Translational Research (ICTR) KL2 program and the facilities and resources at the William S. Middleton Memorial Veterans Hospital. We thank the participants who took part in this study. GRECC manuscript \#2008-33.

Open Access This article is distributed under the terms of the Creative Commons Attribution Noncommercial License which permits any noncommercial use, distribution, and reproduction in any medium, provided the original author(s) and source are credited.

\section{References}

Alexopoulos, G. S. (2005). Depression in the elderly. Lancet, 365 (9475), 1961-1970. doi:10.1016/S0140-6736(05)66665-2.

Alexopoulos, G. S., Gunning-Dixon, F. M., Latoussakis, V., Kanellopoulos, D., \& Murphy, C. F. (2008). Anterior cingulate dysfunction in geriatric depression. International Journal of Geriatric Psychiatry, 23(4), 347-355. doi:10.1002/gps.1939.

Apostolova, L. G., \& Cummings, J. L. (2008). Neuropsychiatric manifestations in mild cognitive impairment: a systematic review of the literature. Dementia and Geriatric Cognitive Disorders, 25 (2), 115-126. doi:10.1159/000112509.

Apostolova, L. G., Dutton, R. A., Dinov, I. D., Hayashi, K. M., Toga, A. W., Cummings, J. L., et al. (2006). Conversion of mild cognitive impairment to Alzheimer disease predicted by hippocampal atrophy maps. Archives of Neurology, 63(5), 693-699. doi:10.1001/archneur.63.5.693.

Ashtari, M., Greenwald, B. S., Kramer-Ginsberg, E., Hu, J., Wu, H., Patel, M., et al. (1999). Hippocampal/amygdala volumes in geriatric depression. Psychological Medicine, 29(3), 629-638. doi:10.1017/S0033291799008405.
Ballmaier, M., Narr, K. L., Toga, A. W., Elderkin-Thompson, V., Thompson, P. M., Hamilton, L., et al. (2008). Hippocampal morphology and distinguishing late-onset from early-onset elderly depression. The American Journal of Psychiatry, 165(2), 229-237. doi:10.1176/appi.ajp. 2007.07030506.

Ballmaier, M., Toga, A. W., Blanton, R. E., Sowell, E. R., Lavretsky, H., Peterson, J., et al. (2004). Anterior cingulate, gyrus rectus, and orbitofrontal abnormalities in elderly depressed patients: an MRI-based parcellation of the prefrontal cortex. The American Journal of Psychiatry, 161(1), 99-108. doi:10.1176/appi.ajp. 161.1.99.

Bell-McGinty, S., Butters, M. A., Meltzer, C. C., Greer, P. J., Reynolds, C. F,. 3rd, \& Becker, J. T. (2002). Brain morphometric abnormalities in geriatric depression: long-term neurobiological effects of illness duration. The American Journal of Psychiatry, 159(8), 1424-1427. doi:10.1176/appi.ajp.159.8.1424.

Bench, C. J., Friston, K. J., Brown, R. G., Scott, L. C., Frackowiak, R. S., \& Dolan, R. J. (1992). The anatomy of melancholia-focal abnormalities of cerebral blood flow in major depression. Psychological Medicine, 22(3), 607-615.

Buckner, R. L. (2004). Memory and executive function in aging and $\mathrm{AD}$ : multiple factors that cause decline and reserve factors that compensate. Neuron, 44(1), 195-208. doi:10.1016/j.neu ron.2004.09.006.

De Asis, J. M., Silbersweig, D. A., Pan, H., Young, R. C., \& Stern, E. (2003). Neuroimaging studies of fronto-limbic dysfunction in geriatric depression. Clinical Neuroscience Research, 2, 324 330. doi:10.1016/S1566-2772(03)00009-4.

Drevets, W. C. (1999). Prefrontal cortical-amygdalar metabolism in major depression. Annals of the New York Academy of Sciences, 877, 614-637. doi:10.1111/j.1749-6632.1999.tb09292.x.

Drevets, W. C., Price, J. L., Simpson, J. R,. Jr, Todd, R. D., Reich, T., Vannier, M., et al. (1997). Subgenual prefrontal cortex abnormalities in mood disorders. Nature, 386(6627), 824-827. doi:10.1038/386824a0.

Fagan, A. M., Csernansky, C. A., Morris, J. C., \& Holtzman, D. M. (2005). The search for antecedent biomarkers of Alzheimer's disease. Journal of Alzheimer's Disease, 8(4), 347-358.

Geda, Y. E., Knopman, D. S., Mrazek, D. A., Jicha, G. A., Smith, G. E., Negash, S., et al. (2006). Depression, apolipoprotein E genotype, and the incidence of mild cognitive impairment: a prospective cohort study. Archives of Neurology, 63(3), 435-440. doi:10.1001/archneur.63.3.435.

Good, C. D., Johnsrude, I. S., Ashburner, J., Henson, R. N., Friston, K. J., \& Frackowiak, R. S. (2001). A voxel-based morphometric study of ageing in 465 normal adult human brains. NeuroImage, 14(1 Pt 1), 21-36. doi:10.1006/nimg.2001.0786.

Greenwald, B.S., Kramer-Ginsberg, E., Bogerts, B., Ashtari, M., Aupperle, P., Wu, H., et al. (1997). Qualitative magnetic resonance imaging findings in geriatric depression. Possible link between lateronset depression and Alzheimer's disease? Psychological Medicine, 27(2), 421-431. doi:10.1017/S0033291796004576

Greicius, M. D., Krasnow, B., Reiss, A. L., \& Menon, V. (2003). Functional connectivity in the resting brain: a network analysis of the default mode hypothesis. Proceedings of the National Academy of Sciences of the United States of America, 100(1), 253-258. doi:10.1073/pnas.0135058100.

Hickie, I., Naismith, S., Ward, P. B., Turner, K., Scott, E., Mitchell, P., et al. (2005). Reduced hippocampal volumes and memory loss in patients with early-and late-onset depression. The British Journal of Psychiatry, 186, 197-202. doi:10.1192/bjp. 186.3.197.

Johnson, S. C., Baxter, L. C., Wilder, L. S., Pipe, J. G., Heiserman, J. E., \& Prigatano, G. P. (2002). Neural correlates of self-reflection. Brain, 125(Pt 8), 1808-1814. doi:10.1093/brain/awf181.

Johnson, S. C., Schmitz, T. W., Trivedi, M. A., Ries, M. L., Torgerson, B. M., Carlsson, C. M., et al. (2006). The influence of Alzheimer 
disease family history and apolipoprotein E epsilon4 on mesial temporal lobe activation. The Journal of Neuroscience, 26(22), 6069-6076. doi:10.1523/JNEUROSCI.0959-06.2006.

Kumar, R., Parslow, R. A., Jorm, A. F., Rosenman, S. J., Maller, J., Meslin, C., et al. (2006). Clinical and neuroimaging correlates of mild cognitive impairment in a middle-aged community sample: the personality and total health through life $60+$ study. Dementia and Geriatric Cognitive Disorders, 21(1), 44-50. doi:10.1159/ 000089251.

Li, Y. S., Meyer, J. S., \& Thornby, J. (2001). Longitudinal follow-up of depressive symptoms among normal versus cognitively impaired elderly. International Journal of Geriatric Psychiatry, 16(7), 718-727. doi:10.1002/gps.423.

Modrego, P. J., \& Ferrandez, J. (2004). Depression in patients with mild cognitive impairment increases the risk of developing dementia of Alzheimer type: a prospective cohort study. Archives of Neurology, 61(8), 1290-1293. doi:10.1001/archneur.61.8.1290.

Morris, R., Paxinos, G., \& Petrides, M. (2000). Architectonic analysis of the human retrosplenial cortex. The Journal of Comparative Neurology, 421(1), 14-28. doi:10.1002/(SICI) 1096-9861 (20000522)421:1\&lt;14::AID-CNE2\&gt;3.0.CO;2-S.

Mueller, S.G., Weiner, M.W., Thal, L.J., Petersen, R.C., Jack, C., Jagust, W., et al. (2005). The Alzheimer's disease neuroimaging initiative. Neuroimaging Clinics of North America, 15(4), 869877. xi-xii. doi:10.1016/j.nic.2005.09.008

Northoff, G., Heinzel, A., de Greck, M., Bermpohl, F., Dobrowolny, H., \& Panksepp, J. (2006). Self-referential processing in our brain-a meta-analysis of imaging studies on the self. NeuroImage, 31(1), 440-457. doi:10.1016/j.neuroimage.2005.12.002.

Qiu, W. Q., Sun, X., Selkoe, D. J., Mwamburi, D. M., Huang, T., Bhadela, R., et al. (2007). Depression is associated with low plasma Abeta42 independently of cardiovascular disease in the homebound elderly. International Journal of Geriatric Psychiatry, 22(6), 536-542. doi:10.1002/gps.1710.

Radloff, L. (1977). The CES-D scale: A self-report depression scale for research in the general population. Applied Psychological Measurement, 1, 385-401. doi:10.1177/014662167700100306.

Raichle, M. E., MacLeod, A. M., Snyder, A. Z., Powers, W. J., Gusnard, D. A., \& Shulman, G. L. (2001). A default mode of brain function. Proceedings of the National Academy of Sciences of the United States of America, 98(2), 676-682. doi:10.1073/pnas.98.2.676.
Rapp, M. A., Schnaider-Beeri, M., Purohit, D. P., Perl, D. P., Haroutunian, V., \& Sano, M. (2008). Increased neurofibrillary tangles in patients with Alzheimer disease with comorbid depression. The American Journal of Geriatric Psychiatry, 16 (2), 168-174. doi:10.1097/JGP.0b013e31816029ec.

Sager, M. A., Hermann, B., \& La Rue, A. (2005). Middle-Aged Children of Persons With Alzheimer's Disease: APOE Genotypes and Cognitive Function in the Wisconsin Registry for Alzheimer's Prevention. Journal of Geriatric Psychiatry and Neurology, 18(4), 245-249. doi:10.1177/0891988705281882.

Shannon, B. J., \& Buckner, R. L. (2004). Functional-anatomic correlates of memory retrieval that suggest nontraditional processing roles for multiple distinct regions within posterior parietal cortex. The Journal of Neuroscience, 24(45), 1008410092. doi:10.1523/JNEUROSCI.2625-04.2004.

Spreen, O., \& Strauss, E. (1998). A compendium of neuropsychological tests: Administration, norms, and commentary (2nd ed.). New York: Oxford University Press.

Steffens, D. C., Byrum, C. E., McQuoid, D. R., Greenberg, D. L., Payne, M. E., Blitchington, T. F., et al. (2000). Hippocampal volume in geriatric depression. Biological Psychiatry, 48(4), 301-309. doi:10.1016/S0006-3223(00)00829-5.

Stepaniuk, J., Ritchie, L., \& Tuokko, H. A. (2008). Neuropsychiatric Impairments as Predictors of Mild Cognitive Impairment, Dementia, and Alzheimer's Disease. Am J Alzheimers Dis Other Demen.

Sun, X., Steffens, D. C., Au, R., Folstein, M., Summergrad, P., Yee, J., et al. (2008). Amyloid-associated depression: a prodromal depression of Alzheimer disease? Archives of General Psychiatry, 65(5), 542-550. doi:10.1001/archpsyc.65.5.542.

Szklo, M., \& Nieto, F. J. (2006). Epidemiology: Beyond the Basics (2nd ed.). Boston: Jones \& Bartlett.

Trivedi, M. A., Wichmann, A. K., Torgerson, B. M., Ward, M. A., Schmitz, T. W., Ries, M. L., et al. (2006). Structural MRI discriminates individuals with mild cognitive impairment from age-matched controls: A combined neuropsychological and voxel based morphometry study. Alzheimer's and Dementia: The Journal of the Alzheimer's Association, 2, 296-302.

Vogt, B. A., Vogt, L., \& Laureys, S. (2006). Cytology and functionally correlated circuits of human posterior cingulate areas. NeuroImage, 29(2), 452-466. doi:10.1016/j.neuro image.2005.07.048. 